\title{
Ultrasonic nondestructive inspection of solid objects
}

\author{
Tadeusz Stepinski \\ Signals and Systems, Uppsala University, Uppsala, Sweden, \\ ts@signal.uu.se
}

\begin{abstract}
Ultrasonic testing (UT) has been used for nondestructive evaluation (NDE) of materials for more than half a century. A number of NDE techniques have been developed and specifi standards have been created for the UT applications in different areas of engineering. UT has two main goals related to inspection of solid objects, firstl, detection and characterization of material discontinuities (fl ws), and secondly, material characterization.

This paper is focused on the selected techniques for $\mathrm{fl} \mathrm{w}$ detection and imaging using bulk waves as well as guided waves. The main part of the paper contains presentation of array techniques used for the inspection of solid structures with focus on ultrasonic imaging of discontinuities. An overview of the high resolution imaging methods based on synthetic aperture concept (SAFT) is given and illustrated with experimental results.

The UT review is concluded with a short presentation of recently developed methods using Lamb waves for monitoring planar structures in structural health monitoring (SHM) applications. Damage detection methods based on application of multiple transducers (2D arrays) for generating and detecting Lamb waves in metallic and composite plates are presented.
\end{abstract}

Keywords: Ultrasound, nondestructive evaluation, ultrasound imaging, synthetic aperture, Lamb waves

PACS: $43.35 . \mathrm{Zc}, 43.38 . \mathrm{Hz}$

\section{INTRODUCTION}

Ultrasonic testing (UT) has been practiced for many decades; already in 1929 the Russian Sokolov proposed to use ultrasound for testing castings. 1949 is the year when the commercial ultrasonic $\mathrm{fl} \mathrm{w}$ detectors designed by Krautkrämer brothers were born. Krautkramer (since 1972 Krautkramer Branson) became world-wide market-leader in the early 60-ies and has kept this position for a number of decennia. Besides Krautkramer new names emerged in the industrial UT: Karl Deutsch and Nukem in Germany, Panametrics and Stavely in USA, Sonatest and Sonomatic in GB, Gilardoni in Italy and Mitsubishi in Japan.

Through the 1980's and continuing until present, computers have provided technicians with smaller and more capable instruments. Computers and robotics enabled motion control that have contributed to the advancement of ultrasonic inspections and replaced manual inspections in many industrial applications. Computerized UT systems can be programmed to inspect large, complex shaped components, with one or multiple transducers or ultrasound arrays collecting data.

Two main setups normally used in NDE for ultrasonic inspection of solid objects are contact and immersion mode. In the contact mode, used commonly in manual inspections, ultrasonic transducer is directly coupled to the surface of the inspected object using a thin layer of contact agent (Fig. 1a). Test result is presented for the operator in the form of an A-scan at the instrument screen. In the immersion mode, shown schematically in Fig. 1b, object and transducer are immersed in a liquid medium (commonly water) and ultrasonic waves propagate towards the inspected object through a thick water layer. In some applications the immersion tank can be replaced with a squirter system, where water jets are used to transmit ultrasound waves to a solid object. To facilitate evaluation of the UT result ultrasound data can be presented in the form of images, B- and C-scans. The resultant C-scan provides a plan or top view of the component. Automatized scanning of components is considerably faster than contact manual inspection and the acoustic coupling is much more consistent.

Ultrasonic inspection has many applications, the most common among them are $\mathrm{fl} \mathrm{w}$ detection/evaluation, dimensional measurements, and material characterization. Mechanized UT provides large data volumes that enable imaging of detected discontinuities/fl ws to evaluate their form and size. There are at least two important factors that are characteristic for UT comparing to medical applications of ultrasound: the majority of inspected objects are solids (mostly metals and composites), and the way how the test results are evaluated. Ultrasound waves propagate in solid with much higher velocity that in water and the detected discontinuities, due to large impedance difference between the parent material and $\mathrm{fl} \mathrm{w}$, take normally the form of hard scatterers. Moreover, spurious echoes are normally observed that 


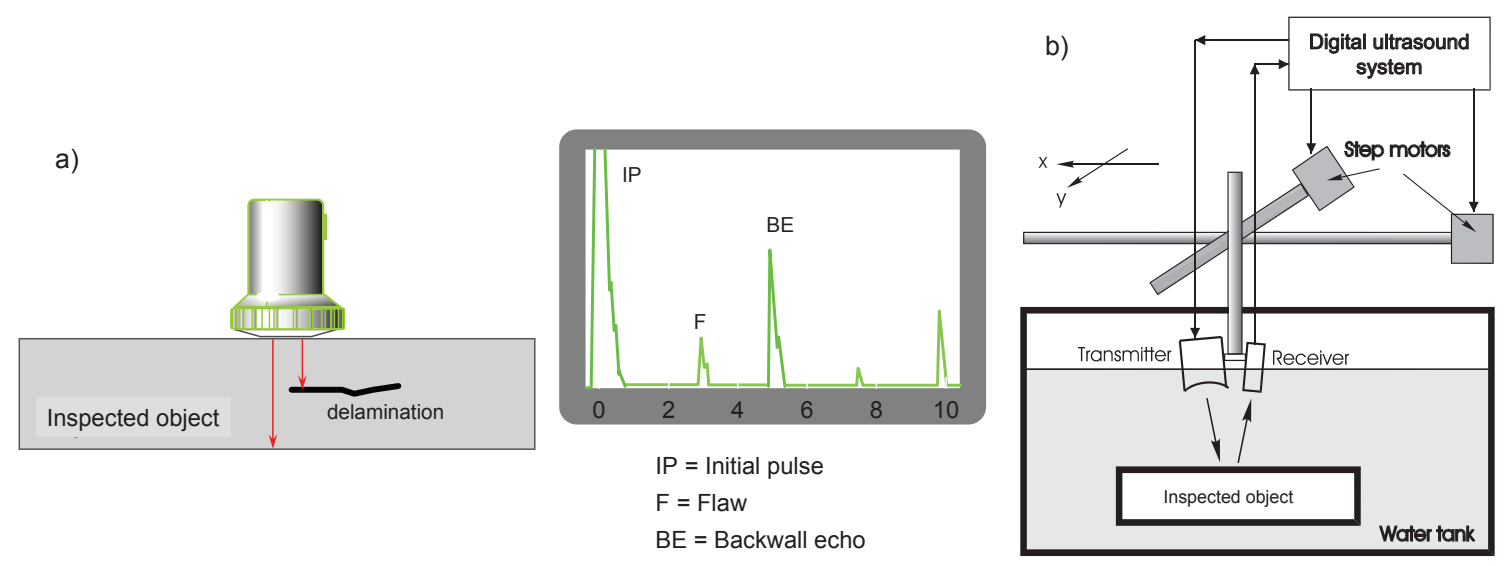

FIGURE 1. The most common test setups used in UT: normal beam transducer in contact mode and instrument screen (a) and $\mathrm{XY}$-scanner used in immersion mode for mechanized inspection (b).

originate from the object geometry, multiple reflection and mode converted waves. Result evaluation is performed to provide a precise quantitative information about detected $\mathrm{fl} \mathrm{ws}$, such as, their size, location and form. The evaluation process is normally carried out based on the operator's expertise and instrument/transducer calibration using special test blocks and mockups. UT operators have to pass special training including both theory and practical exercises on prepared specimens with artificia and natural fl ws.

\section{ULTRASOUND ARRAYS IN NDE}

Phased arrays are relatively new tool that has been introduced to NDE in the recent two decennia. Recent developments of the ultrasonic phased array hardware for NDE enabled a wide use of this technology in industrial applications. One of the most important advantages of phased arrays is their ability to modify beam patterns in an electronic way, that is, the capacity of electronic beam steering and focusing without moving the array or changing its physical components. This feature creates a considerable $\mathrm{fl}$ xibility that accelerates inspecting parts with complex geometry and facilitates the use of UT in many practical applications.

Despite the unquestionable advantages, successful application of phased arrays requires more insight in the mechanism of waves propagation than the conventional ultrasound. Most of the references available in the fiel are concerned with medical applications of phased arrays, which means that they apply to liquid medium only. NDE deals with the detection of hard scatterers in solids and therefore it has different needs from those related to imaging soft scatterers in water.

Modeling tools are needed to design beam patterns of phased arrays with given geometry and focusing law. Array beam patterns are commonly presented in literature for transducers excited with single frequency continuous wave. Real transducers, however, have certain frequency response (bandwidth) that depends on their electromechanical characteristics.

Real arrays have finit sized elements able to emit and receive a finit amount of energy. Element size is mainly limited by the array pitch that determines location of grating lobes. Thus, each array design results from a compromise between the amount of energy emitted/received by its individual elements, and the desired spatial characteristics determining array's spatial resolution as well as the position of grating lobes. Finite sized elements introduce diffraction effects to the array's spatial characteristics that can be clearly observed in the near field

Despite those issues dictating higher requirements for the operators, phased array instruments (PAI) have become commonly used in NDE applications in the past decade. A number of modern portable PAIs have emerged in the market accompanied by specialized arrays both for contact and immersion testing. PHIs, thanks to their fl xibility could considerably accelerate inspections in terms of both data acquisition and result evaluation. Most NDE operators have got used to evaluating images (B- and C-scans) instead of single echoes (A-scans).

As an example one of the firs C-scans acquired in our laboratory in the middle of 90:es is shown in Fig. 2. The inspected object is a section of electron beam (EB) weld from a copper canister to be used for long term storage of 
spent nuclear fuel in Sweden. A number of side drilled and fla bottom holes was drilled in the object to investigate defect detectability in the heat affected zone where a considerable backscattering from the coarse grained material was encountered.

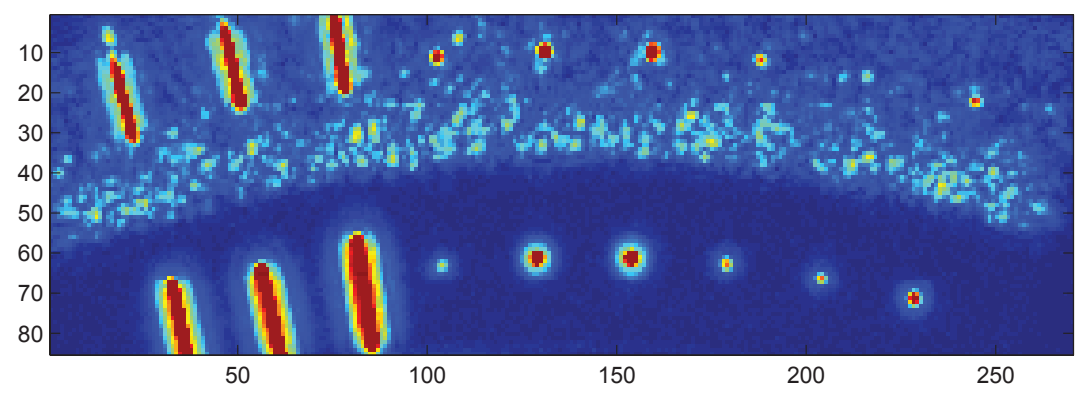

FIGURE 2. C-scan of a copper test block with ultrasonic backscattering from a EB weld clearly visible in the upper part. Side drilled holes well pronounced at the block's left hand side and fla bottom holes pronounced in right right hand side.

\section{Synthetic aperture focusing}

All transducers used for imaging due to their finit aperture introduce distortion in a B-scan image which results in a limited lateral resolution. A considerable work has been done to improve the image quality, which can be categorized in two slightly different approaches: ultrasonic image restoration and synthetic aperture imaging.

The firs approach consists in considering an ultrasonic B-scan as an image distorted by the transducer's point spread function (PSF). If the image distortion can be modeled as a convolution then the deconvolution of the transducer's PSF should result in image restoration. Many authors proposed using the Wiener filte for this purpose $[1,2,3]$.

The second approach, the synthetic aperture focusing technique (SAFT), is based on a concept borrowed from radar imaging techniques. The solution in both application field is to move a small transducer along a line and process the collected data to simulate a desired large aperture. This technique, in its classical time domain realization, consists in delaying each echo signal along the synthetic aperture and coherently summing them to form the image at the focal point. SAFT can also be implemented in the frequency domain using the Fourier transform [4]. Mayer at al. [5] have shown that the Fourier transform SAFT is a special solution of the inverse scattering problem. Furthermore, SAFT being a spatial compounding technique, has several practical advantages. For instance, it can reduce speckle noise and improve the signal-to-noise ratio (SNR).

The conventional time-domain SAFT algorithm performs synthetic focusing by means of coherent summations along hyperbolas in the region of interest (ROI) in the same way as phased array does it for the real aperture in a single focal point. These hyperbolas simply express the distances, or time-delays, from the transducer to the target positions when the transducer is scanned linearly.

There is an important difference, however, between physical linear arrays and synthetic aperture arrays that results in the synthetic aperture having a resolution fine than that corresponding to the real linear array of the same length focused in reception. In the synthetic aperture the same element radiates and receives signals and therefore the roundtrip phase shift is effective in forming the resulting radiation pattern. An important consequence of this fact the synthetic aperture has two times fine cross-range resolution for the same aperture length which, at least in theory, is constant for all points over the ROI $\delta_{3 d B} \cong \frac{d}{2}$, where $d$ is the transducer diameter.

In practical implementations of SAFT imaging there is a trade-off between the size of the transducer and the requirement concerning SNR in the received signal. Using a larger transducer improves the SNR but at the same time introduces a mismatch between the true wave front from the transducer element and the spherical SAFT modeled wave front which reduces the quality of the reconstructed images. 


\section{The model based synthetic aperture imaging}

Most SAFT implementations are based on a very simplifie model of the imaging system used for developing radar and sonar applications. The principal assumption, which is usually correct in radar and sonar, is that the ROI is located in the far fiel of the transducer (antenna) used for creating synthetic array where its specifi diffraction effects can be neglected (point-like source assumption). Unfortunately, this is not always valid in ultrasonic imaging, especially in the high frequency NDE applications where the transducer is often in contact with the inspected structure.

Solution to this problem was proposed in the form of extended SAFT (ESAFT) algorithms that take into account a linear transducer model expressed as a convolution of its spatial impulse response (SIR) with scatterers present in the ROI [6].

An ideal point-like transducer emits a single spherical wave that arrives to the observation point and the associated spatial impulse response (SIR) takes the form of a Dirac pulse. A finit sized transducer has an impulse response which results from the fact that waves emitted by different parts of the transducer arrive at different time instants to an observation point. The SIR will be different for different observation points, that is, contrary to point transducer, its SIR acts as a space dependent (low-pass) filte which results in a degradation of temporal and spatial resolution. The SIRs must, therefore, be accounted for to obtain good performance (high resolution) in ultrasonic imaging when the acoustic waves emitted by the transducer are non-spherical.

Spatiotemporal inverse filter aimed at the deconvolution of transducer SIR require some kind of regularization. The ESAFT algorithm proposed by Lingvall et al [6] is implemented in the time domain and the regularization is derived from the properties of the measurement noise and the a priori knowledge of the scattering targets. The resulting filte , which minimizes the mean squared reconstruction error (MMSE) of the imaging system, has a fi ed structure for a given imaging configuration Thus, the filte can be pre-computed for each imaging configuratio and the fina processing does not require intensive computations.

For illustration we are presenting results of the resolution evaluation performed for the SAFT, MMSE, and phased array (PA) methods in a Rayleigh sense; that is, the ability to distinguish between two closely spaced scatterers. The performance of the methods have been investigated using the phased array system ALLIN and an immersed copper block with four pairs of side drilled holes (SDHs) with a spacing between the holes ranging from 1 to $7 \mathrm{~mm}$ (see Figure 3) [7].

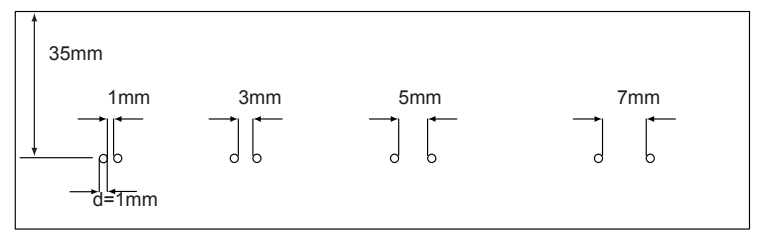

FIGURE 3. Copper Test Block used for resolution comparison.
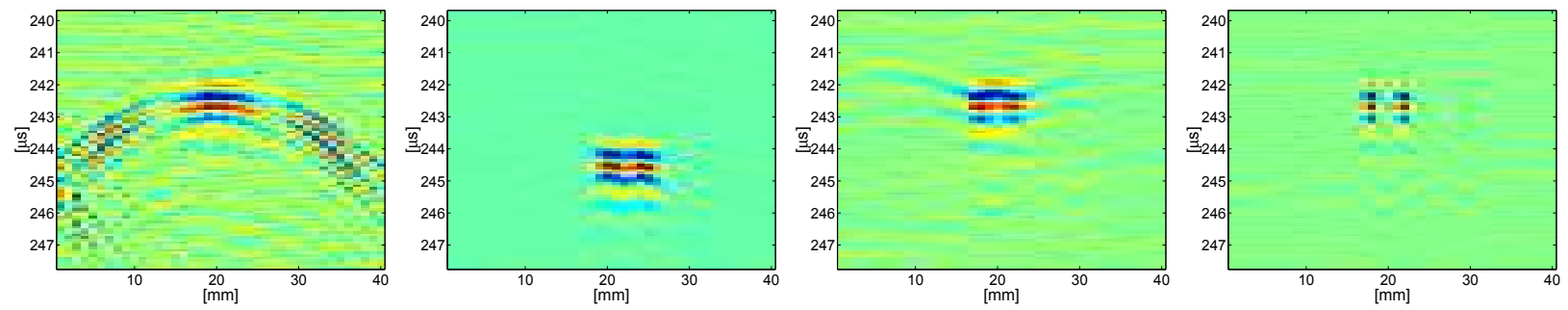

FIGURE 4. Comparison of the resolution obtained using different imaging methods for the holes $5 \mathrm{~mm}$ apart in Fig 3 . From left: original B-scan acquired using $1 \mathrm{~mm}$ element, phased array focused using 32 elements at depth $35 \mathrm{~mm}$ in the block, SAFT and MMSE methods applied to the B-scan acquired using a single $1 \mathrm{~mm}$ element.

A frequency implementation of ESAFT was proposed in [8] in the form of a modifie version of the wavenumber $(\omega-\mathrm{k})$ algorithms known from radar and sonar. The algorithm is derived using a model developed in terms of wave equations. The approximative model, which is valid in the far field accounts for the beam pattern of a finite-size transducer used in the synthetic aperture. Similarly to in the wavenumber implementations, the proposed algorithm employs the 2D FFT for transforming data between the time and frequency domains, and a formal transform of the polar coordinate system, natural for ultrasonic transducers, to the Cartesian system used for the presentation of imaging results. 
Immersion inspection used commonly in NDE creates serious problems for the time domain SAFT due to the refraction of ultrasonic waves at the interface between water and the inspected solid object. For the immersion mode, the ultrasonic waves propagate in two different media (water and solid) and the acoustic path between the transducer and the target is no longer a straight line. The difference between wave velocities in water and the inspected material, such as steel, results in refraction at the interface. According to the Fermat's principle the ultrasonic waves propagate between two points along the path which takes the least time. As shown in Fig. 5, transducer beam is refracted at

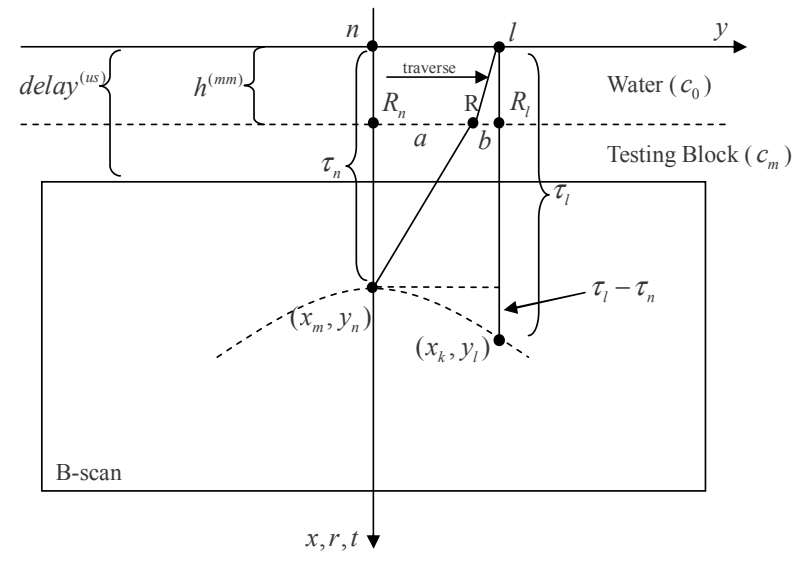

FIGURE 5. Propagating ultrasonic waves in immersion mode. $B$-scan indicates limits of the ROI within the solid test block.

the point $R$ between $R_{n}$ and $R_{l}$ at the boundary between water and solid [9]. To determine the time delay $\tau_{l}$ for each transducer position $\left(0, y_{l}\right)$ and each point within the ROI $\left(x_{m}, y_{n}\right)$ the position of $R$ has to be known (i.e., $a$ and $b$ are to be known). The refraction point $R$ can be determined either using Snell's law or Fermat's principle. Using Snell's law leads to a nonlinear equation, solution of which may be time consuming. A simple search scheme for all candidate points between $R_{l}$ and $R_{n}$ yields the point $R$ with least propagation time according to the Fermat's principle. When the refraction points and the velocities $c_{0}$ and $c_{m}$ are known the respective delays $\tau_{l m n}$ can be calculated using geometrical relations. This method, although conceptually straightforward, results in cumbersome and time consuming calculations in each step $[9,10]$.

\section{The migration approach}

It appears, however, that a scenario with several layers having different sound velocities is an often occurring condition in reflectio seismology where the aim is to estimate the properties of the Earth's subsurface using pulseecho measurements. A specifi approach that is strongly related but not equivalent to synthetic aperture radar and SAFT, called migration, has been developed in seismology. The basic difference between migration and SAFT is that migration makes explicit use of the wave equation in the processing. Viewing the measured signals at the sensors as a boundary condition for the wave equation, the fiel can be extrapolated both forward and backward in time and space. By essentially "turning the clock back", the fiel is back-tracked to the source points.

A considerable advantage of migration approach is that it can be implemented in frequency domain using efficien Fourier transform schemes (FFT). One of those implementations is the phase shift migration method, a frequency domain solution for media that are homogenous in the lateral direction but inhomogeneous in depth, reported in [11]. This approach was adopted to ground penetrating radar (GPR) [12] to solve the problem of different wave propagation velocities in air and soil. Phase shift migration was recently implemented to ultrasound NDE by Olofsson [13, 14]. As an illustration we present experimental results obtained for the 2D phase shift migration applied for processing data acquired in immersion testing of a copper block with a number side drilled holes (SDHs). The immersion test setup used in the experiments is shown in Fig. 6. A $2.25 \mathrm{MHz}$ planar circular transducer from Panametrics with $10 \mathrm{~mm}$ diameter was scanned along the $x$-axis and pulse-echo measurements were acquired at positions $x_{1}, \ldots, x_{210}$ that were separated by $\Delta x=1 \mathrm{~mm}$. The inspected object was placed with its front surface in the horizontal plane. The acquired data is presented in the left panel of Fig. 7 as an envelope B-scan, obtained by Hilbert transforming the raw data. 


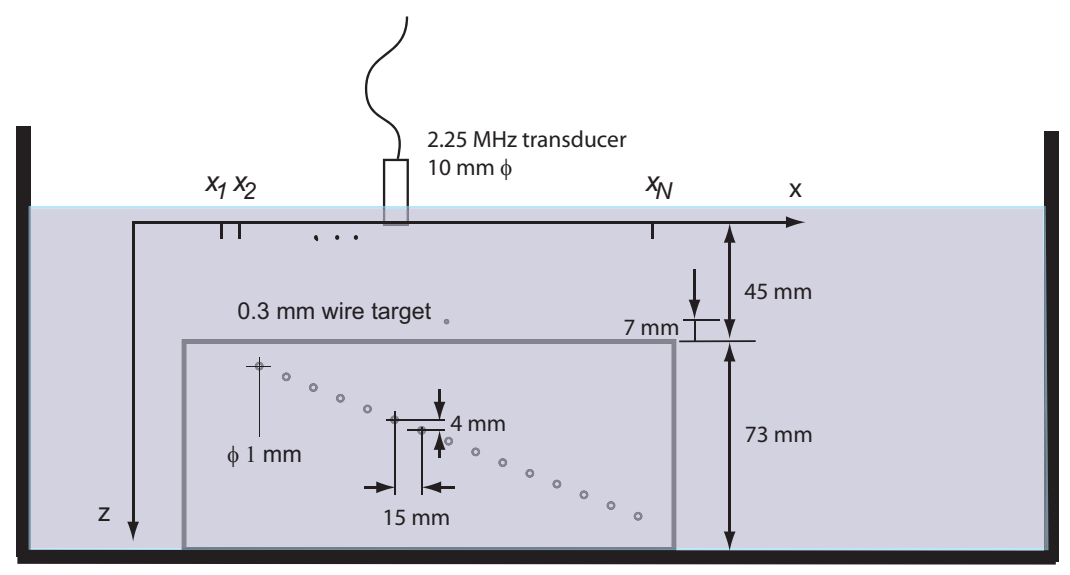

FIGURE 6. Schematic setup for immersion testing of the copper block containing SDHs. The ultrasonic transducer was scanned in the $x$-direction and performed pulse-echo measurements at discrete locations $x_{1}, \ldots, x_{N}$.

The strong front surface echo is seen at approximately $t=60 \mu \mathrm{s}$ corresponding to the water path of approximately $45 \mathrm{~mm}$ and the wire target can be seen at $x=110 \mathrm{~mm}$ at about $t=50 \mu \mathrm{s}$. A secondary echo from the wire is also seen a $t=70 \mu \mathrm{s}$ at the same scanning position. It corresponds to a sound path transducer-Cu-wire-Cu-transducer. The fourteen side drilled holes (SDHs) are seen as hyperbolic patterns at increasing depths.
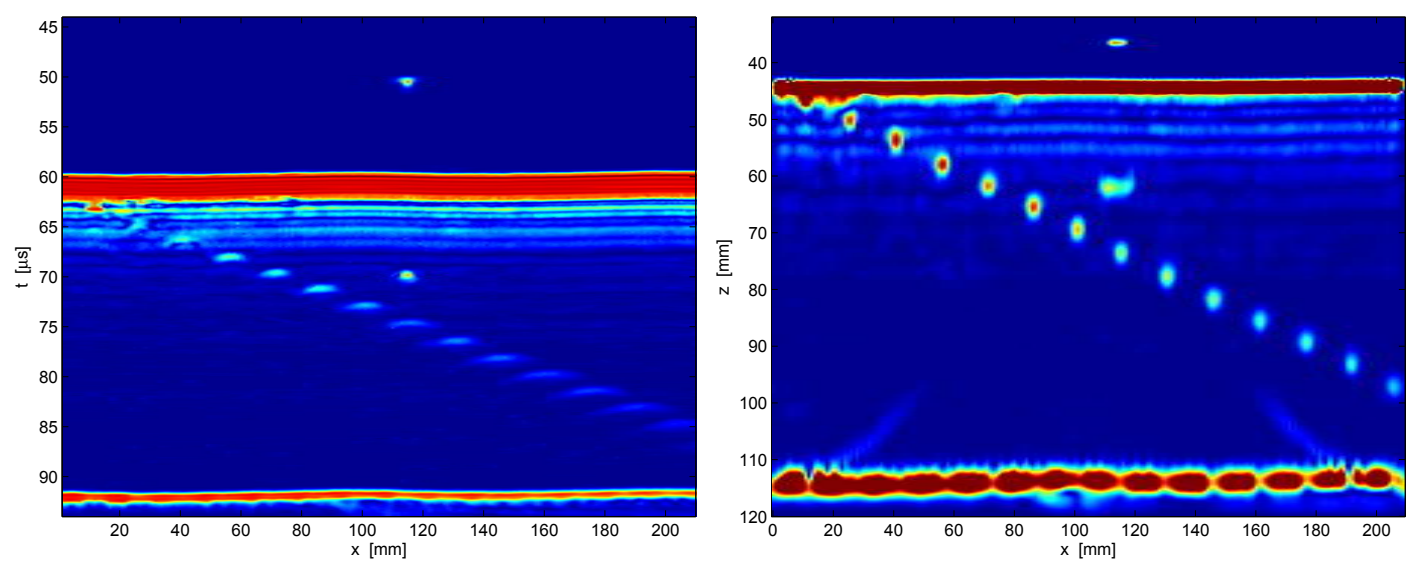

FIGURE 7. The B-scan from the copper block with SDHs and with a wire target in front of the upper surface (left). Image obtained by phase shift migration (right). The hyperbolic patterns representing the SDHs as well as the wire target have been transformed to small spots with lateral resolution that is approximately independent of depth. (Note different scales in the vertical axes: time in $\mu s$, left, and distance in $\mathrm{mm}$, right).

In the migrated image, the responses from the SDHs are shrunk to small spots and the same holds for the wire target. Note that an automatic geometrical correction is obtained through the migration since the method takes into account the different velocities at different layers For example, the wire target's distance between to the front surface of the block can be correctly measured in the image to be $7 \mathrm{~mm}$. Note also that the lateral resolution of the SDHs are approximately equal throughout the entire object.

The diffuse spot centered at $x=110 \mathrm{~mm}$ and $z=61 \mathrm{~mm}$ corresponds the above mentioned double reflectio $\mathrm{Cu}$ wire-Cu. Since multiple reflection are not taken into account in the development of the method, such echoes generally lead to blurred artifacts as the one seen here.

The phase shift migration algorithm presented above was implemented using FFT routines and the current implementation allows processing of the B-scans that takes less time than the data acquisition as well as processing 3D ultrasound data. The phase migration technique requires the knowledge of the sound velocities in the different media involved in the test. Here, only two media were considered, water and copper, but there are no principal restrictions on how many layers we can treat using the method as long as they are all horizontal. It is important to supply the 
migration algorithm with the correct wave velocity values since incorrect values will lead to both poor resolution and poor geometrical correction of the images.

\section{Imaging structures using guided waves}

Guided waves in structures allow inspection of large areas from a fi ed position. Areas which are not accessible using traditional NDE methods can potentially be inspected or monitored using guided waves. Examples of applications for guided waves are the inspection of piping [15] and plates [16].

Guided waves in plates, also known as Lamb waves, share fundamental properties with other types of guided waves, such as, dispersion and the existence of multiple propagating modes. Dispersion causes the signal to spread out which reduces the spatial and temporal resolution of the received unprocessed data, a significan issue in applications where range is to be estimated. Furthermore, Lamb modes differ in dispersion characteristics and propagate at different velocities making interpretation of backscattered signals complicated in the presence of multiple modes [16]. Overlapping modes can make identificatio of small defects and time-of-fligh estimation difficult The effect of dispersion can be reduced by selecting a frequency region with relatively low dispersion or using dispersion compensation scheme.

Dispersion curves of an inspected object can be determined theoretically if the material properties are accurately known and verifie experimentally where a single transmitter/receiver pair or a laser vibrometer provides data allowing the calculation of a spatio-temporal FFT [17].

Below, we present results of an experiment aimed at identifying dispersion characteristics performed on a 6 mm thick aluminum plate (6082-T6). The piezoelectric transmitting transducer was excited by a single square pulse and another transducer was used to sense the propagating Lamb waves in a number of points. Example of the spatiotemporal FFT resulted in a multi-mode signal that can be seen in the frequency-wavenumber power spectrum shown in Fig. 8. From the wave-frequency power spectrum one can identify the modes present in the inspected object and
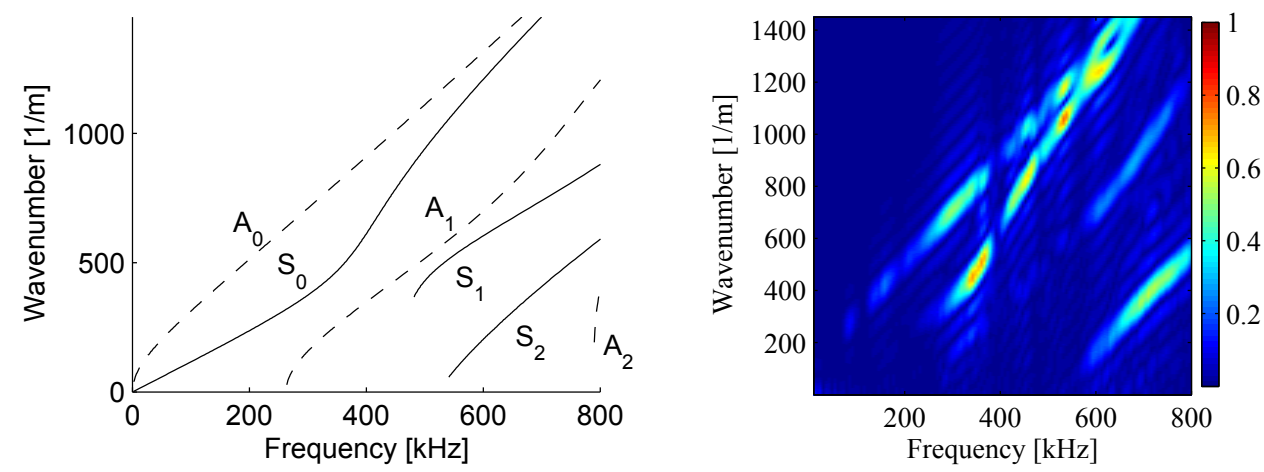

FIGURE 8. Theoretically (a) and experimentally (b) determined dispersion curves for the $6 \mathrm{~mm}$ aluminum plate.

also identify the frequency bands where the interesting wave modes have dominating amplitudes. From Fig. 8 it can be seen that, for instance, the received $S_{0}$ mode dominates the $A_{0}$ mode around $350 \mathrm{kHz}$.

The most commonly reported tool used for imaging discontinues in plates using Lamb waves is standard delayand-sum (DAS) beamformer. The standard beamformer is robust, easy to implement, and computationally simple. However, compared to more advanced array processing methods it suffers from poor resolution and high sidelobes. To improve its performance standard beamformer can be combined with time reversal approach capable of identifying the strongest scatterers in the ROI [18].

Below, we present some experimental results obtained using this approach for the star-shaped fla array shown in Fig. 9b and c. The array contained 32 piezoelectric elements organized in 4 linear sub-arrays (T, A, B and C in Fig. 9b) intersecting at an angle of $45^{\circ}$; each of them consisted of 8 small square PZTs spaced at a distance of $5 \mathrm{~mm}$. The experiments were conducted using the array coupled to the aluminum plate with dimensions $1000 \times 1000 \times 2 \mathrm{~mm}$ shown in Fig. 9a. The plate was provided with three targets simulating damages: a 10x1 mm notch, denoted as $N$, and two small masses $(M 1, M 2)$ that were acoustically coupled to the investigated structure using wax.

In the experiment presented here, 8 array elements in the row $\mathrm{T}$ were used as transmitters while the elements in rows $\mathrm{A}, \mathrm{B}$ and $\mathrm{C}$ as receivers. Imaging consisted of two steps; in the firs step the direction (azimuths) of targets was found experimentally using DORT method (French acronym for decomposition of time-reversal operator), details can 


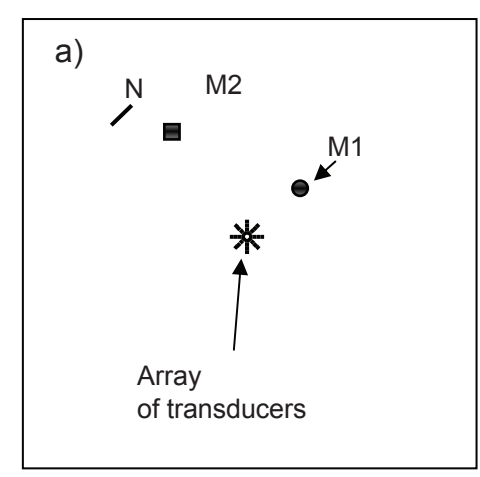

b)

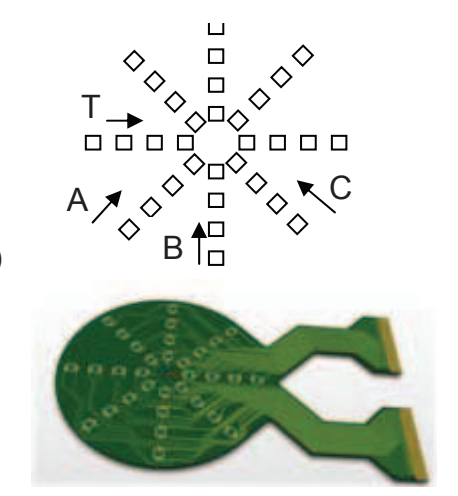

FIGURE 9. Distribution of scatterers in the investigated plate (a). $\mathrm{N}$ denotes notch, M1 and M2 small mass placed on the late. Topology of the star-shaped array (b). Array elements in the row T were used as transmitters while the rows A, B and C as receivers. Laminate board used to build the array $(\mathrm{c})$.

be found in [19]. In the second step the sub-array T was steered sequentially in the detected azimuths of the targets

a)

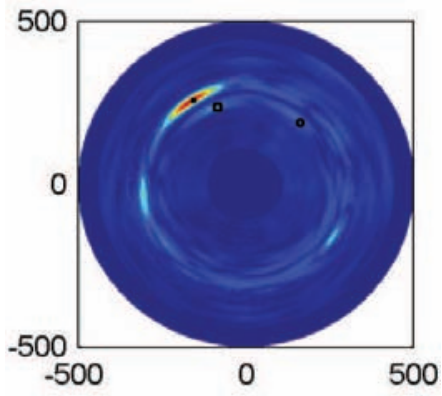

b)

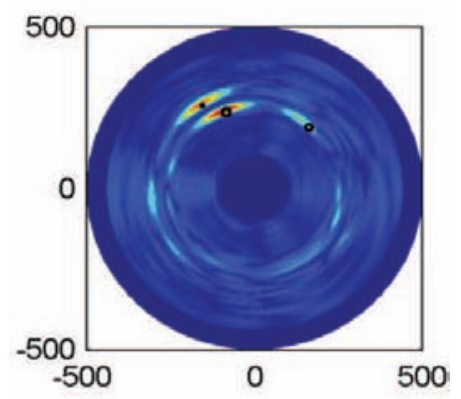

c)

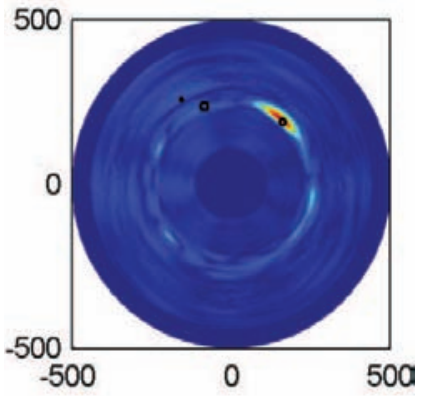

FIGURE 10. Results of damage imaging obtained with the star-shaped array shown in Fig. 9c. Panels a, b and $\mathrm{c}$ show the results for the transmitting sub-array steered in the direction of targets $\mathrm{N}, \mathrm{M} 1$ and M2, respectively.

$\mathrm{N}, \mathrm{M} 1$ and $\mathrm{M} 2$, respectively, and the signals from the sub-arrays $\mathrm{A}, \mathrm{B}$ and $\mathrm{C}$ were acquired for the beamforming in reception. Subsequently, the dispersion removal was applied to the snapshots and the beamforming was performed using DAS scheme. From the results presented in Figs 10 can be seen that the damages were localized correctly. It can be also seen that the 2D topology eliminated mirror images that are normally encountered for linear arrays. Moreover, in all images it can be observed that the illuminated targets are the strongest ones, which proves that the beam azimuths were correctly estimated by DORT in the firs step.

Recently, application of advanced array processing methods based on the theory of adaptive beamforming was proposed for Lamb wave applications [20]. Adaptive beamforming has been successfully utilized in, for example, radar, sonar, and medical ultrasound applications, resulting in higher resolution and better sidelobe suppression compared to the standard DAS beamformer.

The motivation for using this approach is its high resolution and its ability to adaptively suppress interfering signals from other defects and from other propagating Lamb modes. Efficien suppression of interfering modes may relax the requirements on transducer design and limitations in signal bandwidth.

Compared to the DAS beamformer, which is independent of data, an adaptive beamformer is optimized based on some criterion, such as minimizing the influenc of interfering signals in the received data. One of the commonly used adaptive array processing techniques is the minimum variance distortionless response (MVDR) method. A comparison between the MVDR method and a standard beamformer for passive direction-of-arrival (DOA) estimation of Lamb waves, was presented in [21]. Results showed that the MVDR approach outperforms the standard beamformer in terms of resolution and sidelobe level.

The results presented in [22] show that the MVDR beamformer can be very efficien in suppressing interfering 

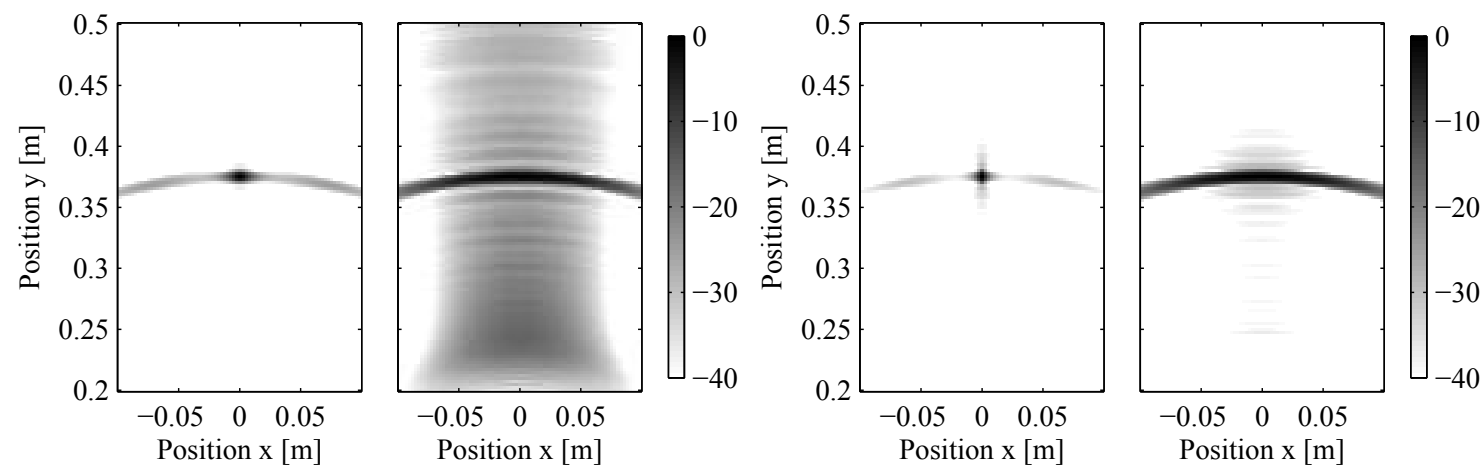

FIGURE 11. Simulated results illustrating the capability of suppressing the $\mathrm{S}_{0}$ mode when focusing on the $A_{0}$ mode of the reflecte $\mathrm{S}_{0}-A_{0}$ modes using using 2x8 (left) and $8 \times 8$ array (right). The MVBF (left) and SBF (right) in each pair.
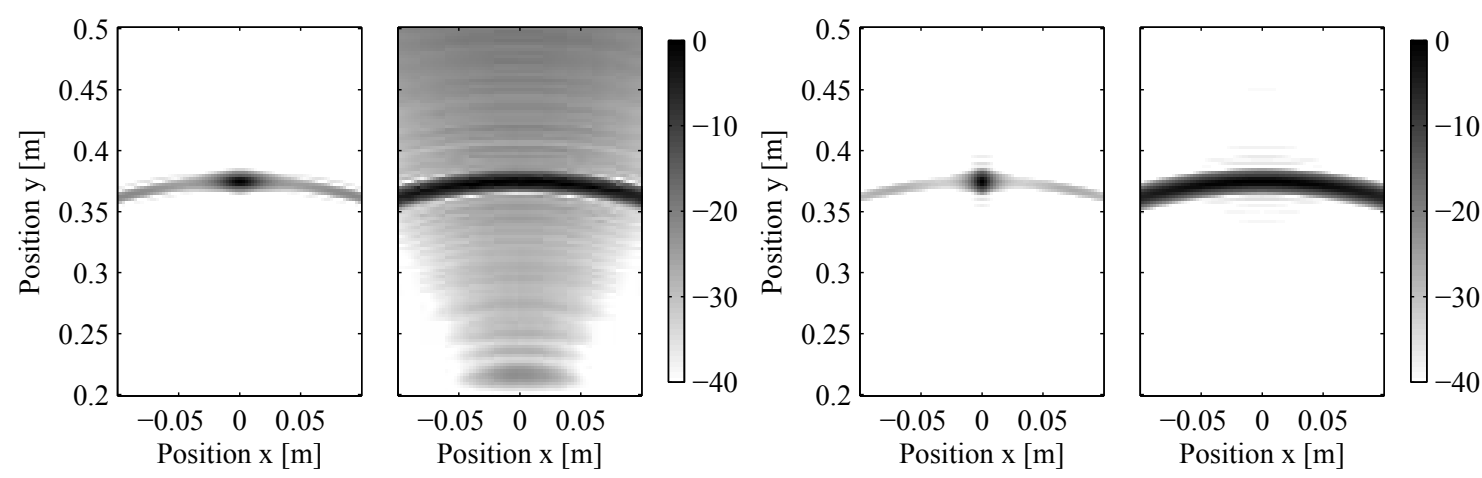

FIGURE 12. Simulated results illustrating the capability of suppressing the $\mathrm{A}_{0}$ mode when focusing on the $S_{0}$ mode of the reflecte $\mathrm{S}_{0}-A_{0}$ modes using using 2x8 (left) and $8 \mathrm{x} 8$ array (right). The MVBF (left) and SBF (right) in each pair.

modes provided that $2 \mathrm{D}$ arrays are used. The authors preformed comparison for rectangular arrays composed of a different number of rows of $8 \times 8$ element array.

In the example shown here, the focusing was performed using the dispersion characteristics of the $\mathrm{A}_{0}$ and $\mathrm{S}_{0}$ modes. Two Lamb modes were simulated, the $\mathrm{A}_{0}$ and $\mathrm{S}_{0}$ modes, which were reflecte from a single reflecto located $375 \mathrm{~mm}$ from the center of the $8 \times 8$ array. Both modes were reflecte with unit reflectio coefficient The element spacing was approximately half the minimum wavelength of the $\mathrm{A}_{0}$ mode in the frequency range used. The input signal was a 1 cycle $300 \mathrm{kHz}$ sinusoid which was bandpass filtere to a bandwidth of $260 \mathrm{kHz}$. All array elements were excited sequentially and the back-scattered signals were calculated for each array element. The results obtained using two rows of $8 \times 8$ array and the full $8 \times 8$ array, when focusing on the $A_{0}$ and $S_{0}$ mode, are presented in Figs 11 and 12, respectively. In the frequency range of the simulated signals, the $\mathrm{A}_{0}$ mode is only slightly dispersive contrary to the $\mathrm{S}_{0}$ mode, which is highly dispersive. This can be seen in Fig. 11 where the $\mathrm{S}_{0}$ mode is smeared over a wide range. In Fig. 12 the dispersion compensation of the $\mathrm{S}_{0}$ has the reverse effect on the $\mathrm{A}_{0}$ mode, it becomes dispersed. From Figs 11 and 12 can be seen that also in terms of angular resolution, the MVDR clearly outperforms the standard DAS beamformer.

\section{SUMMARY AND CONCLUSION}

Ultrasonic nondestructive inspection of structures, although based on the same physical principles as medical ultrasound and sonar, has developed its own application specifi techniques. The main goal of UT is detecting and characterizing $\mathrm{fl} \mathrm{ws}$ in solid object that are normally stationary during the test. The task is complicated, however, by the fact that the small hard scattering discontinuities present in those objects are to be detected and characterized in presence 
of geometrical reflections multiple echoes and mode converted reflections

This paper gives a selective overview of modern techniques used for ultrasonic testing of objects and structures in industrial applications. The focus was on the use of real and virtual arrays of ultrasound transducers. It was shown how different techniques invented for other applications, such as, synthetic aperture radar and migration of geophysical data can be used for imaging discontinuities in solid objects.

It was also shown that beamforming of guided waves in planar structures requires more sophisticated means that that used for bulk ultrasonic waves. Specialized sensor arrays and techniques have been developed in this fiel which is a part of dynamically developing area of structural health monitoring (SHM).

\section{ACKNOWLEDGMENTS}

Most of the results presented here have been obtained as a result of team work at Uppsala University in the projects supported by SKB (the Swedish Nuclear Fuel and Waste Management Co) which is gratefully acknowledged. This paper would not be possible without the contributions of my my former PhD students: M. Engholm, F. Lingvall and T. Olofsson. Research funding from the Polish research project MONIT (No. POIG.01.01.02-00-013/08-00) concerned with SHM and the contributions of the research team from the AGH University of Science and Technology in Kraków, Poland are also acknowledged.

\section{REFERENCES}

1. M. Fatemi, and A. Kak, Ultrasonic Imaging 2, 1-47 (1980).

2. W. Vollmann, IEEE Trans. on Sonics and Ultrasonics 29, 78-83 (1982).

3. D. Iraca, L. Landini, and L. Verrazzani, IEEE Trans. on Ultrasonics, Ferroelectrics, and Frequency Control 36, 216-222 (1989).

4. K. Nagai, IEEE Trans. on Sonics and Ultrasonics 32, 531-536 (1985).

5. K. Mayer, R. Marklein, K. Langenberg, and T. Kreutter, Ultrasonics 28, 241-255 (1990).

6. F. Lingvall, T. Olofsson, and T. Stepinski, J. Acoust. Soc. Am. 114, 225-234 (2003).

7. T. Stepinski, F. Lingvall, and P. Wu, Inspection of copper canisters for spent nuclear fuel by means of ultrasound, Tech. Rep. TR-03-05, SKB (2002).

8. T. Stepinski, IEEE Transactions on Acoustics, Speech, and Signal Processing 54, 1399-1408 (2007).

9. T. Stepinski, Synthetic aperture focusing technique in ultrasonic inspection of coarse grained materials, Tech. rep., SKI Report 2008:06 (2008).

10. T. Stepinski, and F. Lingvall, "Synthetic aperture focusing techniques for ultrasonic imaging of solid objects," in EUSAR2010 -8th European Conference on Synthetic Aperture Radar, 2010.

11. J. Gazdag, Geophysics 43, 1342-1351 (1978).

12. K. Gu, G. Wang, and J. Li, IEE Proceedings: Radar, Sonar and Navigation 151, 317-325 (2004).

13. T. Stepinski (editor), M. Engholm, and T. Olofsson, Inspection of copper canisters for spent nuclear fuel by means of ultrasound, Tech. Rep. TR-08-12, SKB (2008).

14. T. Olofsson, IEEE Trans. on Ultrasonics, Ferroelectronics and Frequency Control 57, 2522-2530 (2010).

15. W. Mohr, and P. Höller, IEEE Transactions on Sonics and Ultrasonics 23, 369-374 (1976).

16. A. Raghavan, and C. Cesnik, Shock and Vibration Digest 39, 91 - 114 (2007), ISSN 0583-1024.

17. D. Alleyne, and P. Cawley, Journal of the Acoustical Society of America 89, 1159 - 1168 (1991), ISSN 0001-4966.

18. L. Ambrozinski, T. Stepinski, P. Packo, and T. Uhl, accepted for Mechanical Systems and Signal Processing (2011).

19. L. Ambrozinski, T. Stepinski, and T. Uhl, "Self focusing of 2D arrays for SHM of plate-like structures using time reversal operator.," in International Workshop on Structural Health Monitoring 2011 (accepted), 2011.

20. M. Engholm, and T. Stepinski, Ultrasonics, Ferroelectrics and Frequency Control, IEEE Transactions on 57, 2712-24 (2010),

21. T. Stepinski, and M. Engholm, "Piezoelectric Circular Array for Structural Health Monitoring Using Plate Waves," in 7th International Workshop on Structural Health Monitoring, Stanford, CA USA, 2009.

22. M. Engholm, T. Stepinski, and T. Olofsson, accepted for Smart Materials and Structures (2011). 